$$
\text { CONF-970159--Z }
$$

\title{
THREE NEW NONDESTRUCTIVE EVALUATION TOOLS BASED ON HIGH FLUX NEUTRON SOURCES
}

Camden R. Hubbard*, Dudley Raine, Roberta Peascoe, Michael Wright, Paul M. Whaley, and Paul Koehler, Oak Ridge National Laboratory, Oak Ridge, TN 37831-6064 (ph: 423-574 4472; e-mail: hubbardcr@ornl.gov)

\section{ABSTRACT}

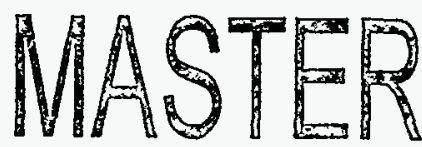

Nondestructive evaluation methods and systems based on specific attributes of neutron interactions with materials are being developed. The special attributes of neutrons are low attenuation in most engineering materials, strong interaction with low $\mathrm{Z}$ elements, and epithermal neutron absorption resonances. The three methods under development at ORNL include neutron based tomography and radiography; through thickness, nondestructive texture mapping; and internal, noninvasive temperature measurement. All three techniques require. high flux sources such as the High Flux Isotope Reactor, a steady state source, or the Oak Ridge Electron Linear Accelerator, a pulsed neutron source. Neutrons are quite penetrating in most engineering materials and thus can be useful to detect internal flaws and features. Hydrogen atoms, such as in a hydrocarbon fuel, lubricant, or a metal hydride, are relatively opaque to neutron transmission and thus neutron based tomography/radiography is ideal to image their presence. Texture, the nonrandom orientation of crystalline grains within materials, can be mapped nondestructively using neutron diffraction methods. Epithermal neutron resonance absorption is being studied as a noncontacting temperature sensor. This paper highlights the underlying physics of the methods, progress in development, and the potential benefits for science and industry of the three facilities.

\section{INTERACTION OF NEUTRONS WITH MATERIALS}

Neutron interactions with matter are different from $x$-ray interactions and as such can be used in complementary or unique ways for nondestructive characterization of materials. $\mathrm{X}$-ray interactions are dominated by the photoelectric effect and are thus highly dependent on the atomic number of the element. On the other hand, the interaction probability for a neutron and a nucleus, derived from quantum mechanics, requires only that some part of the neutron wave interact with the nucleus of the atom. The result is that while the interaction probability for $x$-rays generally increases steadily with the atomic number of the target atom, no periodic relationship governs the resulting interaction probability for a neutron. Neighboring elements (and different isotopes of the same element) can have very different neutron interaction probabilities. Nearly all interaction mechanisms for neutrons lead to attenuation of the incident beam.

Table I compares the attenuation of thermal neutrons with that for $125 \mathrm{keV} x$-rays. Note the high attenuation coefficient for low $\mathrm{Z}$ elements, particularly for hydrogen. Most engineering materials have lower attenuation for neutrons than for $125 \mathrm{keV} x$-rays and hence by using neutrons it is possible to image larger components and simultaneously detect small quantities of hydrogen within the material. This characteristic of neutron interactions means that neutron radiography and computed tomography can be used alone or in conjunction with $\mathrm{x}$-ray techniques to obtain nondestructive images or tomographs of a material that would be difficult or impossible to acquire otherwise.

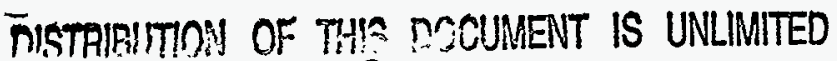

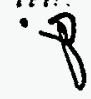

\footnotetext{
"The submitted manuscript has been authored by a contractor of the U.S. Government under contract No. DE-AC05-96OR22464. Accordingly, the U.S. Government retains a nonexclusive, royalty-free license to publish or reproduce the published form of this contribution, or allow others to do so, for U.S. Government purposes."
} 


\section{DISCLAIMER}

Portions of this document may be illegible in electronic image products. Images are produced from the best available original document. 
Table I. Comparison of Attenuation Coefficients for Neutrons and High Energy X-rays

\begin{tabular}{|c|c|c|c|c|c|c|c|c|}
\hline & $H$ & $D$ & $L i$ & $A L$ & $F e$ & $Z r$ & $\bar{W}$ & $\bar{U}$ \\
\hline $\begin{array}{l}\text { Attenuation Coefficient } \\
\text { Thermal Neutrons } \\
\left(\mathrm{cm}^{2} / \mathrm{g}\right)\{1\}\end{array}$ & 22.8 & $\overline{0.41}$ & $\overline{6.28}$ & 0.037 & 0.147 & 0.054 & 0.079 & 0.040 \\
\hline $\begin{array}{l}\text { Attenuation Coefficient } \\
125 \mathrm{keV} \mathrm{X} \text {-rays } \\
\left(\mathrm{cm}^{2} / \mathrm{g}\right)(2\}\end{array}$ & 0.279 & .0 .279 & 0.128 & 0.150 & 0.253 & 0.564 & 2.51 & 4.04 \\
\hline
\end{tabular}

Table II compares the attenuation of neutrons with a $1.0 \AA$ wavelength to that of $\mathrm{Cu} K \alpha$ characteristic $x$-rays typically used for diffraction studies. In the case of diffracted neutrons, the cross section of true absorption is unimportant compared to the scattering cross section for most materials. In table II, the total cross sections collected by Hughs and Schwartz\{3\} are used to calculate $\mathrm{d}_{1 / 2}$. Here, the thermal neutrons have penetration depths of several hundreds to thousands of times that of the characteristic x-ray of similar wavelength. Thermal neutrons are particularly attractive for studies of a wide range of engineering materials including iron, aluminum, alumina, and many others.

Table II. Comparison of Penetration Depths for Thermal Neutrons and $\mathrm{Cu} \mathrm{K} \alpha \mathrm{X}$-rays

\begin{tabular}{l|rrr}
\hline Material & $d_{1 / 2} X$-rays $(\mu \mathrm{m})$ & $d_{1 / 2}$ Neutrons $(\mu \mathrm{m})$ & Penetration \\
& $\lambda=1.5418 \AA(\mathrm{CuK \alpha})$ & $\lambda=1.0 \AA$ & ratio \\
\hline Aluminum & 52.8 & 76760 & 1454 \\
Titanium & 10.7 & 16113 & 1506 \\
Iron & 2.9 & 7841 & 2703 \\
Copper & 14.6 & 8545 & 585 \\
$\mathrm{Al}_{2} \mathrm{O}_{3}$ & 56.1 & 18590 & 331 \\
$\mathrm{SiO}_{2}$ & 75.9 & 24300 & 320 \\
$\mathrm{Fe}_{3} \mathrm{O}_{4}$ & 5.9 & 9290 & 1574 \\
\hline
\end{tabular}

$d_{1 / 2}$ is the penetration depth at which the intensity of the incident beam is reduced by $50 \%$. The penetration ratio is $d_{1 / 2}$ (neutrons) to $d_{1 / 2}$ ( $x$-rays).

\section{NEUTRON RADIOGRAPHY AND COMPUTED TOMOGRAPHY}

The basic imaging techniques of neutron radiography and $\mathrm{x}$-ray radiography are similar. An object is placed between a source and a recording medium and an image is formed by the transmitted radiation. The difference in the measured intensity at any point in the image plane reflects the effects of the attenuation properties along a corresponding path through the object. However, constructing a facility for neutron radiography is much more difficult than for $\mathrm{x}$-ray radiography and requires special considerations during development.

Factors that affect the overall. quality of the neutron images include the neutron flux, LD ratio, thermal neutron to gamma ratio, flux uniformity, and imaging method. A high flux neutron beam has a greater dynamic range and penetrating ability for most applications. The $L D$ ratio is the ratio of the length of the collimator $(L)$ to the diameter of the aperture (D). The L/D ratio gives some information about the angular divergence of the beam and the inherent image unsharpness. The thermal neutron to gamma ratio is an indicator of the inherent contrast of an image, since gamma rays in the beam will be attenuated differently from the neutrons and most imaging mediums have some sensitivity to gamma rays. The flux intensity across the beam should also vary by as small an amount as possible. The imaging method, as with $\mathrm{x}$-rays, is an 
important consideration since there is an inherent unsharpness in the resulting image from the detection process, and detection materials respond in different ways depending on the energy spectrum of the incident neutrons.

The beam port being installed at the High Flux Isotope Reactor.(HFIR) is expected to result in one of the highest quality neutron beams in the world. The unfiltered neutron flux has been calculated using Monte Carlo methods to be approximately $4 \times 10^{9} \mathrm{n} / \mathrm{cm}^{2} \cdot \mathrm{s}$ with an effective $L / D$ ratio between 75 and 125 . The high flux also makes the beam a good candidate for applications involving real-time radiography, where dynamic processes can be observed. The high flux coupled with new, high speed neutron detectors will enable routine neutron tomography where numerous data sets are required.

Neutron Computed Tomography (NCT) is analogous to $x$-ray $C T$, which both the industrial and medical communities use for many applications. Neutron tomography is different from neutron radiography in that the resulting image is a two-dimensional cross section through the object obtained by reconstruction algorithms. As with neutron radiography, the facility characteristics play a major role in the quality of the collected data. In NCT the detector configuration is particularly important as it determines the resolution of the resulting reconstructions and must have a high count rate capability for a large number of pixels.

The new beam tube at HFIR will be installed in spring, 1997, for development of a prototype radiography and tomography system. Studies are currently underway to optimize the beam filter to reduce the gamma content in the beam without significantly affecting the neutron flux. ASTM standards will be used to determine the effective beam parameters. For film radiography, a thin $\mathrm{Gd}$ foil in a film pack will be used. Gd converts thermal neutrons into low energy internal conversion electrons that the film records. For tomography applications, a prototype detector based on ${ }^{6} \mathrm{Li} / \mathrm{ZnS}$ phosphor, fiber optics, a fast photomultiplier and application specific integrated circuits is currently under development and being tested. This detector will have a resolution of $1 \mathrm{~mm}^{2}$ and a count rate capability of $>10^{6} \mathrm{~Hz}$.

There are many applications for both neutron radiography and computed tomography. The primary use for neutron radiography in industry is the nondestructive detection of corrosion and/or manufacturing defects. The military maintains a facility at McClellan AFB with the primary purpose of detecting corrosion in the wings of military aircraft. The nuclear power industry also uses neutron radiography and computed tomography to evaluate the integrity of reactor components. Other applications abound for neutron techniques and include void and inclusion detection in castings and weldments, hydrocarbon location, detection of hydrogen embrittlement, and studies of water migration and two-phase (boiling) flow.

\section{THROUGH THICKNESS, NON DESTRUCTIVE TEXTURE MAPPING}

The nonrandom distribution of crystallites or grains in a polycrystalline specimen is called texture. Texture ranges from the extremes of a single crystal to a statistically random distribution of a fine grained polycrystalline material. Diffraction methods are used to quantitatively describe texture via pole figures and the orientation distribution function. A pole figure is a graphical representation of the ratio of the diffracted intensity from a crystallographic set of hkl planes for all orientations of the specimen with respect to a specimen with a completely random grain distribution. Because material properties can be directionally dependent, the properties in a given direction of a textured specimen can differ from the bulk average property. This can be true for thermal expansion, hardness, electrical conductivity, thermal diffusivity, and in fact, nearly every material property. Some materials engineers utilize texture to achieve properties not possible with conventional polycrystalline materials. One striking example is the effort to achieve extensive 
texture in high Tc superconducting materials because electrical conductivity is far higher in the a-b plane than perpendicular to the plane.

$\mathrm{X}$-ray diffraction methods $\{4\}$ are used to measure the texture of the material within a few microns of the surface of a specimen. Depth mapping (through thickness) using $x$-rays requires destructive sectioning that can introduce changes in the surface, thus requiring extensive specimen preparation including etching or electro polishing. The gain in depth penetration of thermal neutrons compared to $x$-rays used for diffraction (Table II) has several advantages: (1) using neutrons permits bulk average measurements free of the surface conditions; (2) whereas $\mathrm{x}$-rays sample only a few surface grains, larger volumes can be sampled by neutrons and the results will be free of coarse microstructure effects. The coarse microstructure effect is a significant problem for $\mathrm{x}$-ray methods with many metals and alloys.

Another advantage of neutron diffraction is that neutron scattering factors can be substantially different from $x$-ray scattering factors. This difference can lead to measurable changes in the diffraction pattern of neutrons and $x$-rays. For example, figure 1 shows the pattern for alpha- $\mathrm{Al}_{2} \mathrm{O}_{3}$ for neutrons and $\mathrm{x}$-rays of the same wavelength. Note that the basal plane reflection (006) is only observed in the neutron diffraction pattern. This reflection was used by Brokmeier\{5\} in a study of the thermal shock behavior of copper-bonded alumina substrates. The texture analysis indicated that a random texture was advantageous for improving the thermal shock behavior. The neutron diffraction method was shown to be a rapid, sensitive tool to assess texture in $\mathrm{Al}_{2} \mathrm{O}_{3}$.

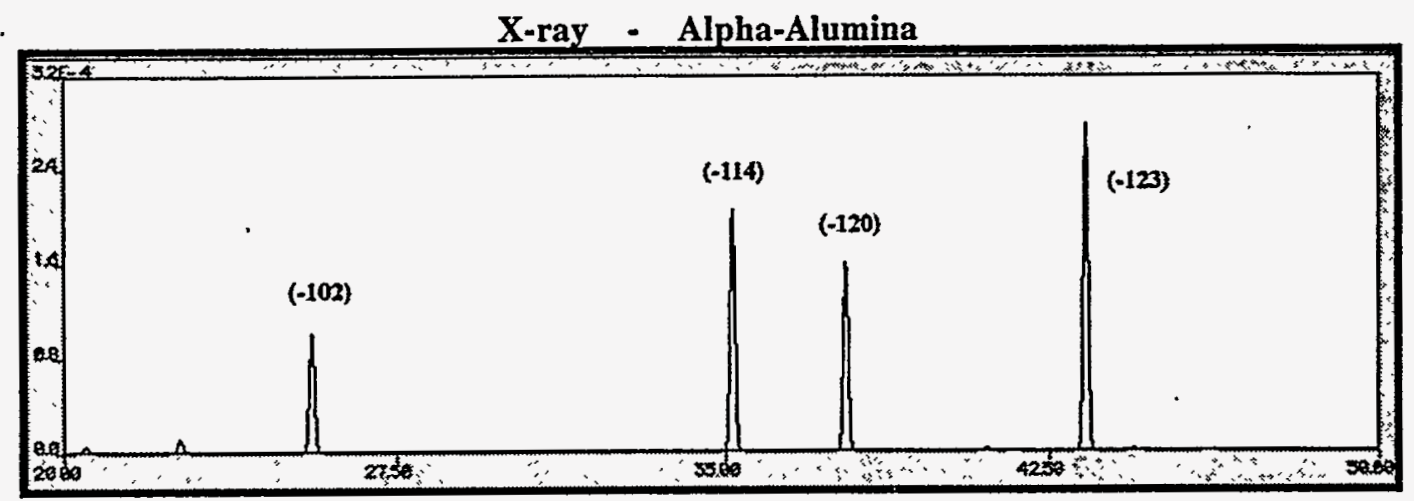

Neutron - Alpha-Alumina

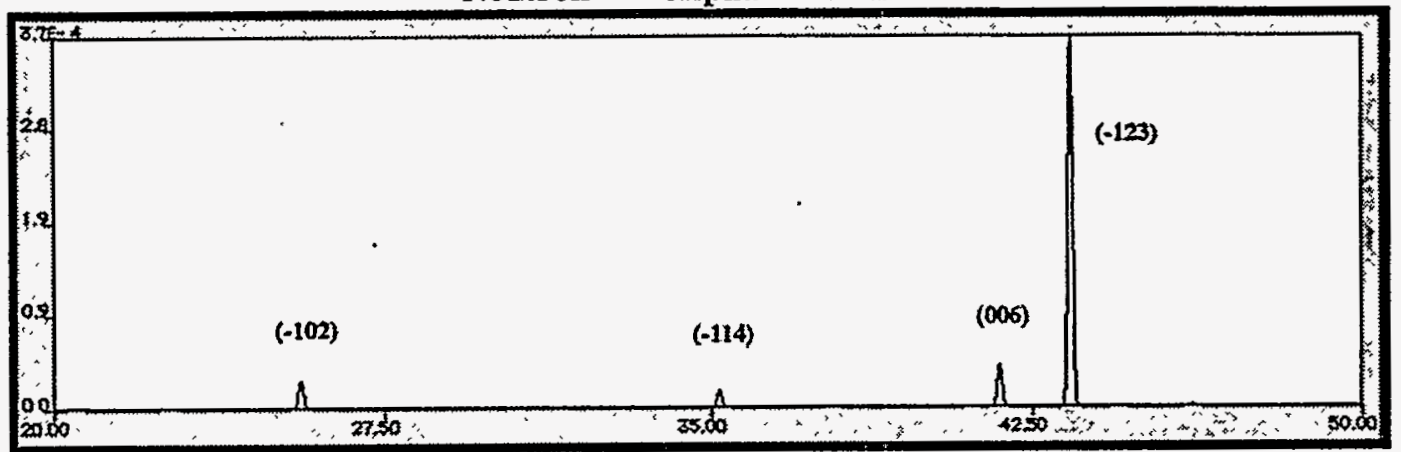

Figure 1. X-ray and neutron diffraction patterns calculated for alpha-alumina with $\lambda=1.514 \AA$. 
Developments at ORNL aim to establish a nondestructive neutron diffraction texture mapping facility for use by US industry, academic and laboratory scientists and engineers. The facility currently under development will be located at the HFIR and take advantage of its high flux. To further reduce measurement times, a novel ${ }^{6} \mathrm{Li} / \mathrm{ZnS}$ based $256 \times 256$ position sensitive neutron detector is being developed that has a $17 \times 17 \mathrm{~cm}$ active area. With this 2-D detector, sections of a pole figure can be collected simultaneously, and in some cases two or more hkl's at a time, leading to major gains in the speed of data collection.

We will also establish a through thickness mapping capability where the size of the sampling volume within the specimen is defined by incident and receiving beam slits (Figure 2), much as it is done in through thickness residual stress mapping\{6\}. Brokmeier recently used sectioning to characterize the changes in texture of an aluminum ingot at the edge and mid-width $\{3\}$. He used neutron diffraction methods to overcome the large grain size problem that prevented use of $x$-ray methods and revealed that the texture changed from fully random at the mid-width to a $<100\rangle$ fiber texture at the edge of the ingot. Neutron texture analysis methods are being used to assist the manufacturer to control texture inhomogeneity and improve the product. The goal for the ORNL facility at HFIR is to provide rapid, nonintrusive, through thickness mapping of texture in a broad range of engineering and research materials. Such a facility will offer the potential to observe texture evolution at multiple locations in a single specimen. Observations following each processing step, for example, after sintering, hot pressing, rolling, swaging, or forging, can be performed using the nondestructive neutron diffraction method. One use of texture studies will be to validate finite element materials process models, for example, of castings.

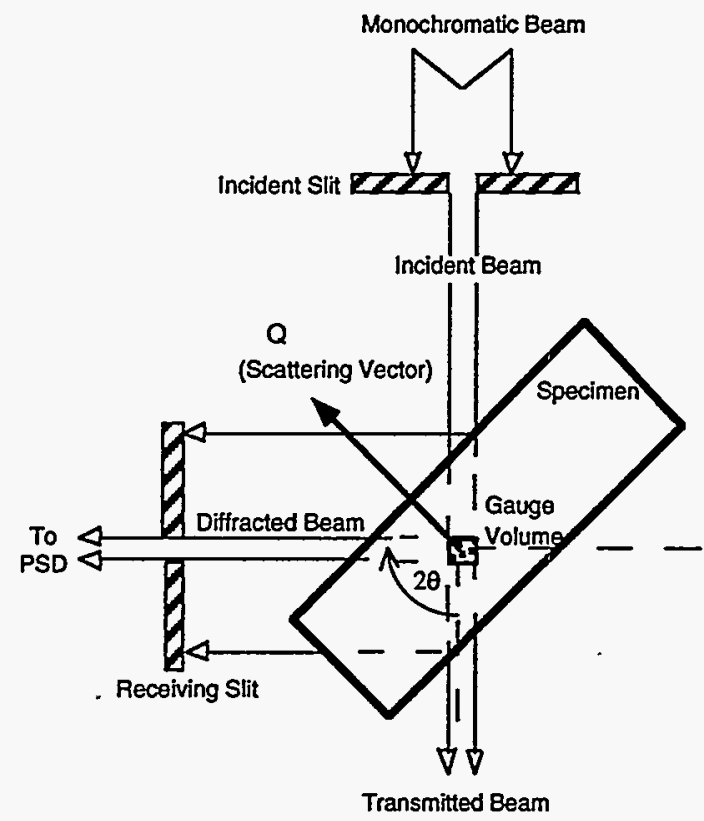

Figure 2. Scheme for mapping residual stresses at an arbitrary location within a specimen. For texture mapping, the 1-D position sensitive detector (PSD) will be replaced by a 2-D PSD.

A recent study at HFIR using the existing 1-D position sensitive detector shows the improvements in pole figure data obtainable using neutron diffraction instead of $\mathbf{x}$-ray diffraction. For this study, a test specimen was prepared by bonding seven layers of rolled Fe sheet metal to 
make a specimen of about $1 \mathrm{~cm}^{3}$. The rolling direction of each layer was coincident. The measurement of each pole figure was performed with monochromatic neutrons $(\lambda=1.5 \AA)$ at the HB2 beam line. The (110) and (200) pole figures were recorded and compared to data from the same specimen collected on an $\mathrm{x}$-ray texture goniometer. The unsmoothed data in Figure 3 show that the expected symmetry is most clearly visible in the neutron pole figure. The $\mathrm{x}$-ray data show considerable noise and lack of symmetry, likely due to the low number of grains in the x-ray diffracting volume. Another advantage of the neutron technique is that the data collection included tilts of the specimen to $90^{\circ}$ while the $\mathrm{x}$-ray data was truncated at $80^{\circ}$ due to absorption issues when the sample is highly inclined with respect to the beam. The full coverage by neutrons is a considerable advantage when one wishes to determine the orientation distribution function from multiple pole figures. Approximation methods used to extend the $\mathrm{x}$-ray information beyond the maximum tilt angle are unnecessary in neutron texture analysis.

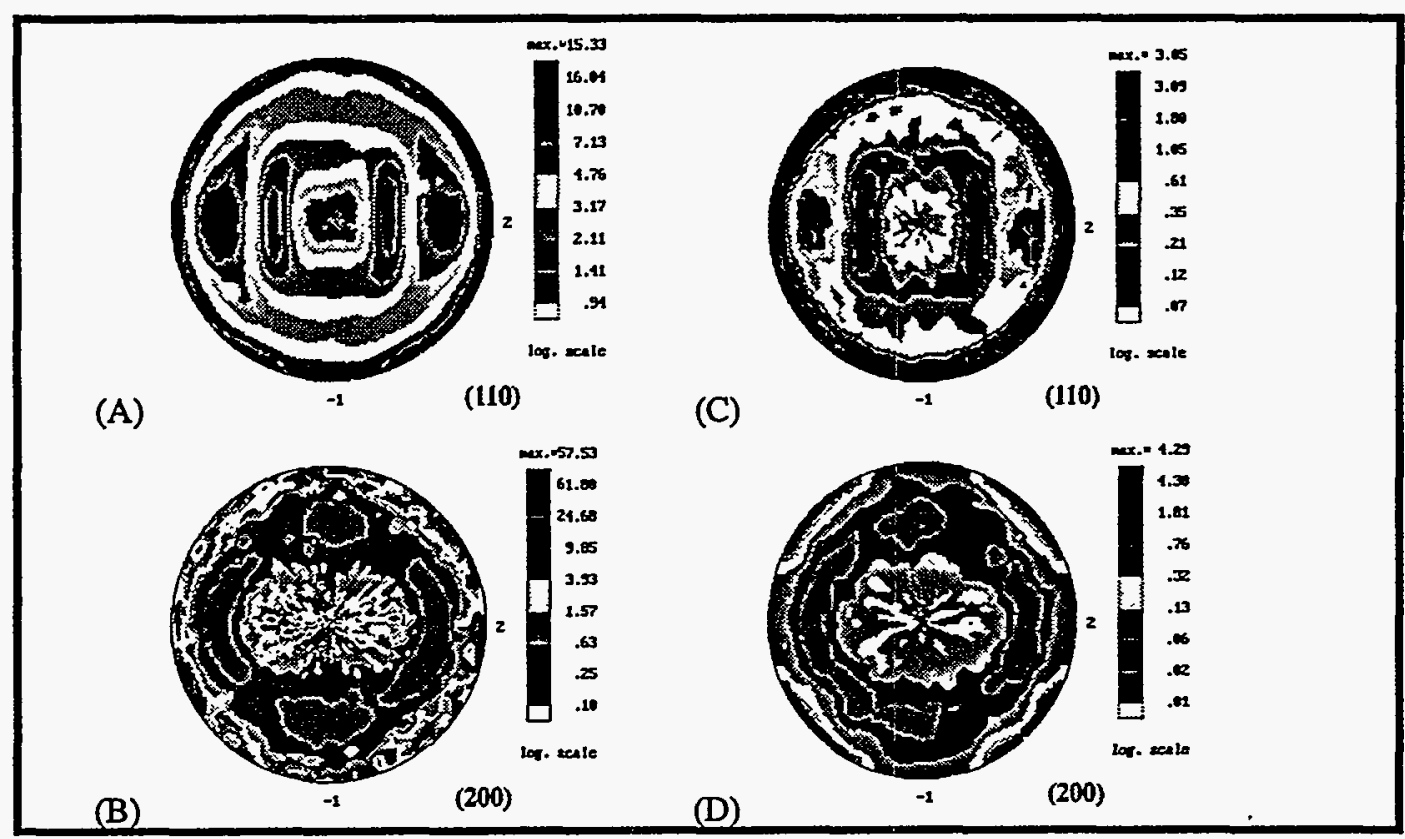

Figure 3. The (110) and (200) pole figures of rolled Fe sheet metal for bulk sample using neutron diffraction ( $A$ and $B$ ) and the etched surface using $x$-ray diffraction $(C$ and $D)$.

\section{IN SITU NEUTRON-BASED TEMPERATURE MEASUREMENT}

The feasibility of measuring temperature remotely and noninvasively with epithermal neutrons and the Doppler broadening properties of absorption resonances in the $1<\mathrm{E}<100 \mathrm{eV}$ energy range has been presented by Fowler and Taylor $\{7\}$, Haste and Sowerby $\{8\}$, and Stewart and Fowler $\{9\}$. A number of elements, particularly those with high $Z$, have useful epithermal absorption resonances including, $\mathrm{Ta}, \mathrm{Au}, \mathrm{Hf}, \mathrm{Re}, \mathrm{Rh}, \mathrm{Pt}, \mathrm{Sm}, \mathrm{Ag}, \mathrm{Tm}$, and $\mathrm{W}$. Thermal motion of the element increases with increasing temperature and results in a Doppler broadening of the absorption resonance (Figure 4). To better reveal the Doppler broadening, the difference in the cross section between $600 \mathrm{~K}$ and $400 \mathrm{~K}$ over the ${ }^{181} \mathrm{Ta}$ resonance is plotted (Figure 5). This difference displays a "crater-like" plot that, when normalized to unity, gives a ratio of neutron counts between the two exposures. This ratio is used to determine the temperature of the bulk material. The reported sensitivity to temperature measurement is better than $1^{\circ} \mathrm{C}$ for $\mathrm{Ta}$ at $1000^{\circ} \mathrm{C}$. 
Fowler and Taylor have suggested many improvements to the basic method involving innovative. detectors, similar to the one discussed above for neutron tomography.

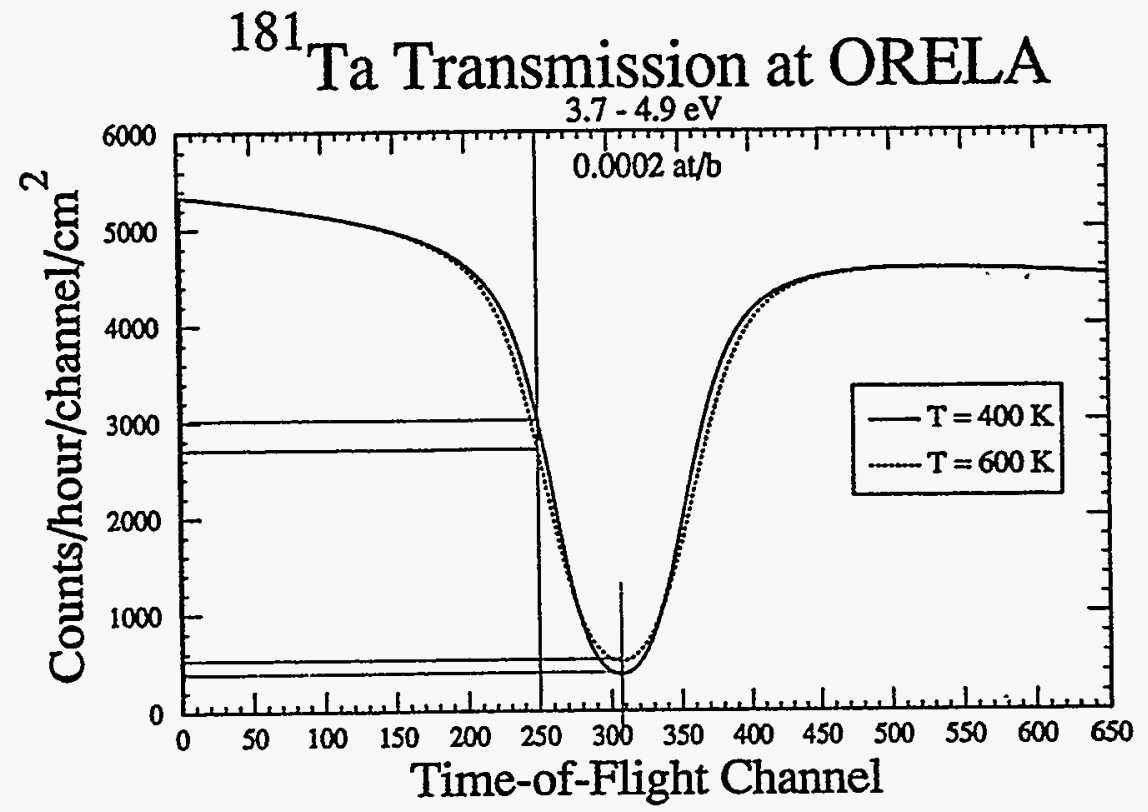

Figure 4. Calculated profiles for epithermal neutron absorption by a thin coating of Ta for two temperatures showing an increased in the width of the absorption resonance with a $200 \mathrm{~K}$ change in temperature.

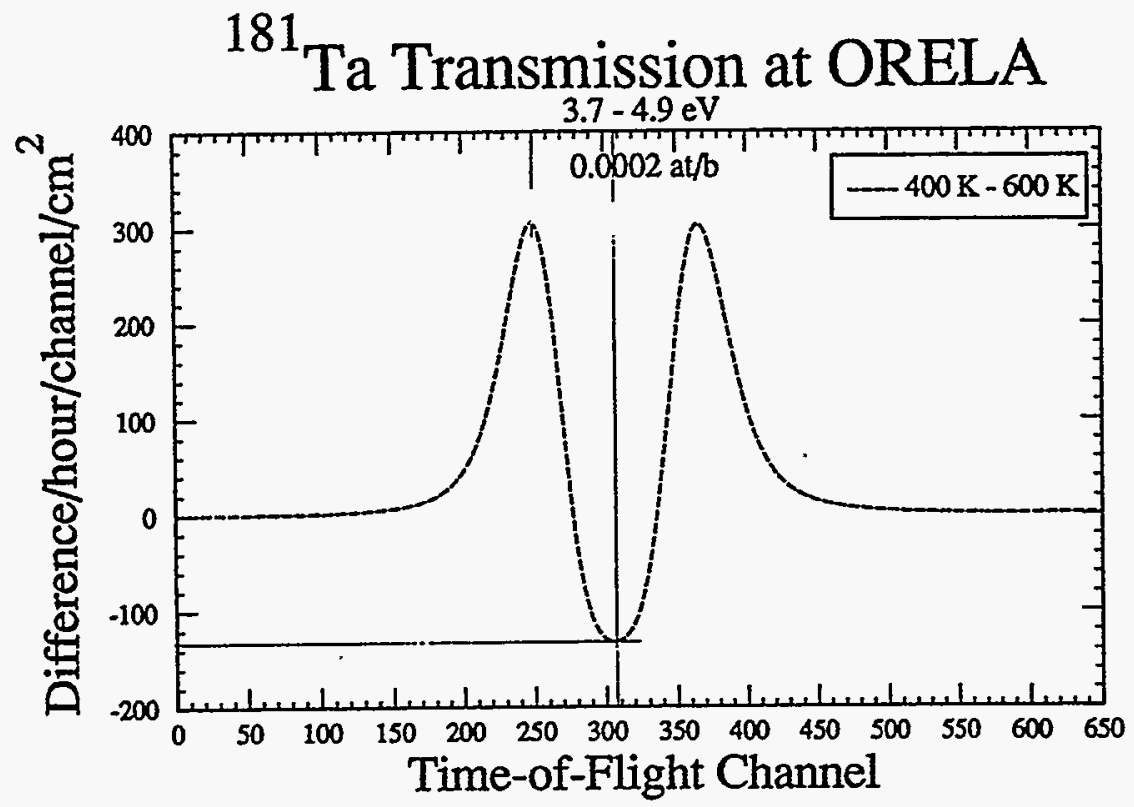

Figure 5. Plot of the difference in counts for the calculated profiles in Figure 3 for a Ta foil at $400 \mathrm{~K}$ and $600 \mathrm{~K}$ at the $4.28 \mathrm{eV}^{181} \mathrm{Ta}$ resonance. 
The noninvasive temperature measurement method is being assessed at ORNL with the goal of determining its practical limits at the existing Oak Ridge Electron Linear Accelerator (ORELA), which is a moderate flux pulsed source, and the proposed National Spallation Neutron Source. The spectra shown in Figures 4 and 5 are the predicted results from a $20 \mu \mathrm{m}$ thick Ta layer $1 \mathrm{~cm}^{2}$ collected in 1 hour. The technique has considerable potential for measurements that are impractical using other methods. For example, surfaces can be coated with selected thin metallic patches to determine the temperature of the surface in an operating engine. With 2-D detectors it will be possible to measure temperature gradients over large areas at the same time, which would be impractical with thermocouples. The pulsed nature of the epithermal neutron source can be used as a stroboscopic measurement tool by synchronizing the beam pulse with a rotating or reciprocating component such as a piston or turbine.

\section{REFERENCES}

1. "Reactor Physics Constants," 2nd ed., Argonne National Laboratory, Report ANL-5800 (1963) 30 .

2. National Nuclear Data Center Online Data Service (http://www.nndc.bnl.gov), Brookhaven National Laboratory, (1991).

3. Hughes, D. J., Schwarts, R. B., BNL-Report 325 (1958).

4. Bunge, H. J. in H.J. Bunge, E. Esling (eds.), Advances and Applications of Quantitative Texture Analysis, DGM-Informationsgesellschaft, Oberursel (1991) 241-278.

5. Brokmeier, H. G., "Neutron Diffraction Texture Analysis for Industrial Applications", Z. Metallkd. 85 (1994) 8, 598-602.

6. Wang, X.-L., Hubbard, C.R., Spooner, S., David, S.A., Rabin, B.H., and Williamson, R.L., "Mapping of the residual stress distribution in a brazed zirconia-iron joint", Materials Science and Engineering A211 (1996) 45-53.

7. Fowler,P.H. and Taylor, A.D., "Temperature Imaging Using Epithermal Neutrons", Rutherford Appleton Lab., Publication RAL-87-056 (August 1987).

8. Haste, T.J. and Sowerby, M.G.; "A study of the temperature dependence of the neutron transmission of uranium dioxide", J. Phys. D: Appl. Phys., Vol. 12 (1979) 1203-1221.

9. Stewart, P.A.E. and Fowler, P.H; "Temperature measuring", US patent 4,839,519 (1989).

\section{ACKNOWLEDGMENT}

This research was sponsored by the ORNL Laboratory Directed Research and Development Fund. ORNL is managed by Lockheed Martin Energy Research Corp. for the U.S. Department of Energy under contract number DE-AC05 96OR22464.

\section{DISCLAIMER}

This report was prepared as an account of work sponsored by an agency of the United States Government. Neither the United States Government nor any agency thereof, nor any of their employees, makes any warranty, express or implied, or assumes any legal liability or responsibility for the accuracy, completeness, or usefulness of any information, apparatus, product, or process disclosed, or represents that its use would not infringe privately owned rights. Reference herein to any specific commercial product; process, or service by trade name, trademark, manufacturer, or otherwise does not necessarily constitute or imply its endorsement, recommendation, or favoring by the United States Government or any agency thereof. The views and opinions of authors expressed herein do not necessarily state or reflect those of the United States Government or any agency thereof. 